\title{
Bulvar and psevdobulbar syndrome
}

\author{
Boboyorov Sardor Uchqun o'g'li ${ }^{1}$ \\ ${ }^{I}$ Termez branch of the Tashkent Medical Academy \\ Stage 3 "Treatment" faculty a student of the 309th group in the field of "Medical work" \\ ( sardorbekboboyorov@gmail.com )
}

\begin{abstract}
This article provides recommendations for the treatment of boulevard and pseudobulbar syndromes, which are relatively rare in our lives, which are caused by nerve damage.

Keywords: Bulbar syndrome, dysarthria, dysphagia, central nervous system disorders.

\section{INTRODUCTION}

Bulbar syndrome or bulbar paralysis. It is a syndrome caused by damage to the bulbar nerves located in the cerebellum, the main symptoms of which are dysphagia (swallowing disorder), dysarthria (speech articulation disorder) and dysphonia (presentation of vocal cords).

Topography. The cerebral cortex includes the cerebral cortex, the Varoli bridge, and the cerebellum. Bulbar palsy is observed in the lower part of the brainstem, ie in the nucleus and roots of the nerves IX (tongue-throat), X (planetary) and XII (tongue) located in the elongated brain. Bulbus is an old name for the elongated brain.

Etiology. Diseases that damage the brainstem and bulbar nerves cause bulbar palsy. These are the pillars of the brain stroke, edema, encephalitis, syringobulbia, amyotrophic lateral sclerosis, diphtheria, myasthenia gravis, Guillain-Barré syndrome, botulism and others. and the vocal cords are paralyzed. Therefore, bulbar syndrome is also called boulevard paralysis. This paralysis is peripheral paralysis.
\end{abstract}

\section{MAIN PART}

The patient's voice is reduced, he speaks in a hushed voice. Such a patient is reminiscent of a person with a strong cold. The patient does not have difficulty chewing food (because the $\mathrm{V}$ nerve innervates the chewing muscles), but has difficulty swallowing it. Especially when swallowing liquid food, it comes back through the nose. It is known that the larynx is an anatomical structure located at the border of the airways and the upper part of the esophagus. The muscles of the larynx and the structures around it play a key role in pushing food into the esophagus. In bulbar paralysis, this process is disrupted, resulting in the risk of food debris getting into the airways. If this condition is observed frequently, there is a risk of developing pneumonia. The diagnostic value of neurological examinations is great. To diagnose dysarthria and dysphonia in a patient, it is sufficient to filter it. He speaks in a low voice, unable to pronounce phonetic and consonant phrases. When the patient is asked to say the $\langle\mathrm{A} \gg$ sound louder, his voice is uta sunik; or the sound does not come out. Attention is also paid to the condition of the soft palate: it hangs on the paralyzed side, and on the healthy side it shrinks and turns to the side of the tongue. When examined with a spatula, the swallowing reflex on the paralyzed side is not induced or decreased. When the patient is asked to remove the tongue, atrophy and fascicular vibrations are detected in half of it, the tongue tilts in this direction. Tongue fasciitis is also called a "chewing tongue" symptom by neurologists. To diagnose dysphagia, the patient is given a small amount of water in a teaspoon. At this point, the patient becomes unable to swallow water and begins to cough. Water should be carefully placed on the front of the mouth. Depending on the etiology of the disease, bulbar paralysis manifests itself to varying degrees. Sometimes it goes light. Acutely developed bulbar palsy (e.g., Guillain-Barré syndrome) is life-threatening and can be fatal. UAVs that detect bulbar palsy in a patient should be referred to a neurologist immediately. Pseudobulbar syndrome or pseudobulbar paralysis. The main symptoms of this syndrome are dysarthria, dysphagia and dysphonia. Therefore, the topic of bulbar and pseudobulbar syndromes is side by side in neurology textbooks.

Etiology and topography. Bulbar syndrome develops due to damage to both sides of the corticobulbar tract, which innervates the IX, X and XII nerves. As a result, the bulbar function, that is, the functions of the tongue and throat, is lost. Corticobulbar (corticonuclear) pathways are central pyramidal pathways that begin in the motor nuclei of the cerebral hemispheres and reach the bulbar nerves. Therefore, pseudobulbar palsy is one of the central paralysis. For the development of pseudobulbar syndrome, the corticobulbar ducts must be damaged on both sides. If these pathways are damaged on one side, pseudobulbar syndrome will not develop. Thus, strokes, degenerative diseases, tumors, hematomas and other large processes that develop in both 
hemispheres of the brain lead to the development of pseudobulbar syndrome.

Clinic. It consists of dysarthria, dysphagia and dysphonia, ie the patient has impaired swallowing and speech, a sharp decrease in voice, slurred speech. In contrast to bulbar syndrome, the swallowing reflex is maintained or increased, atrophy and fasciculations on the tongue are not observed.

Reflexes of loud crying and laughing and oral automatism appear. To check this reflex, apply a neurological hammer or finger to the patient's upper and lower lips; is built by tapping. In this case, the patient strengthens the lips forward, creating a situation similar to the upic movement. The lower jaw reflex is also

Treatment. As these syndromes develop due to various diseases, treatment is carried out depending on the etiology of the disease Atypical prosopalgia (atypical facial pain).

Atypical prosopalgias are persistent pain and paresthesias that occur on one or both sides of the face. The disease occurs mainly in the age group of 30-60 years. The disease is more common in women. Atypical prosopalgias usually result from dental surgery. Such pains are more common in people who have had their upper and lower jaw teeth changed and prostheses burned on them. Although the clinic of atypical prosopalgia is similar to the clinic of cranial neuralgia or neuropathy, objective neurological symptoms (hyperesthesia, hypoesthesia) are not detected. There are no trigger areas on the face. Organic disorders of the nerves are not detected during in-depth examination of dental sites, as well as during ENMG examinations. Pain and paresthesias are observed not only at the site of surgery, but also in the facial area of the face. Short-term pain attacks are not typical for the disease, the pain is worse, worse, worse. Atypical prosopalgia is also called idiopathic facial pain.

Another clinical type of atypical prosopalgia is atypical odontalgia. Pain that persists for a long time at the site of tooth extraction is called atypical odontalgia. Even in atypical odontalgia, objective neurological symptoms are not detected. This syndrome is more common in patients with tooth decay and burns or in longterm dental procedures. Pain at the site of tooth extraction and irradiation to other teeth persists for up to a month, sometimes longer. The clinic of atypical odontalgia is similar to the clinic of dental plexalgia, but it is milder than it is.

Dental plexalgia is observed when the nerve endings of the upper and lower jaws are affected. The main cause of dental plexalgia is dental treatments performed on the upper and lower jaw teeth. Pain is manifested along with vegetative symptoms, because the nerve endings here are closely connected with the vegetative ganglia. Although dental plexalgia causes facial pain, ascites, and dysesthesia, no objective neurological symptoms (hypoesthesia, hyperesthesia) are bserved. Dental plexalgia usually lasts for months, sometimes more than a year. Prolonged pain leads to reactive neurotic disorders. It is important to calm the patient and reassure him that these symptoms are temporary. If the doctor can do it, the disease will pass in 1-2 months.

Horton's syndrome (Chakka arteritis). The temporal artery is one of the branches of the external carotid artery (a.carotis externa). Chakka arteritis is an autoimmune disease that mainly occurs in people over 50 years of age. Although the clinical picture of temporal arteritis is similar to that of trigeminal neuralgia, it has many different clinical and paraclinical symptoms.

The main symptom of temporal arteritis is severe pain in the temples and forehead. However, these pains are often observed on both sides. Chronic pain sometimes intensifies and becomes unbearable. Pain often worsens when chewing food. Objective examinations show thickening and thickening of the temporal artery. When the artery is very constricted, its beating is not noticeable. Localized pain also spreads to other parts of the head. Body temperature also rises to subfebrile levels, profuse sweating and general weakness are observed. The patient quickly becomes tired. As temporal arteritis is a common autoimmune disease, lesions are also found in the arteries of the body, and in most cases there is damage to the internal carotid artery, aorta, and coronary arteries. Retinal artery damage is manifested by decreased vision. There is also damage to the optic nerve and the nerves that drive the fall. the number of inflammatory reactions, ie leukocytosis, ECG and an increase in the amount of S-reactive protein. Duplex scanning and carotid angiography are widely used in the diagnosis of temporal arteritis. A temporal artery biopsy is performed to clarify the diagnosis. Hunt syndrome (knee joint neuralgia). Inside the fallopian tube, the XIII nerve fibers (sensory and autonomic) that run along the facial nerve change direction and form the knee ganglion \{ganglion geniculi). Knee nodal neuralgia develops from various viral infections. Painful and burning pain is observed in the anterior part of the supraspinatus, the external auditory canal, hard palate, kshchori jaw and sliding tumor. Strong stinging pain is manifested by taste disturbances and hypersalivation in the anterior two-thirds of the tongue. If knee hernia neuralgia is caused by the herpes zoster virus, herpetic rashes will spread to the areas where the burning pain is observed. This is called Hunt syndrome. Occasionally, mild paralysis of the facial nerve is observed. When 
the virus infects several surrounding ganglia, the painful and herpetic rash spreads to the face, forehead, temples, neck and neck, increases salivation, nystagmus and dizziness. This type of herpetic neuralgia is severe and lasts a long time. Talm op le giya syndrome of pain (Tolos-Hunt syndrome). This syndrome develops in granulematous inflammation of the outer wall of the cavernous sinus and the upper lobe of the fall bowl. The pathological process involves several cranial nerves, ie the nerves that move the fall, the first branch of the trigeminal nerve, and sometimes the optic nerve. Almost always the sympathetic ligaments of the internal carotid artery are damaged. The disease is manifested by "burning", burning and irritating pains in the autumn bowl, ophthalmoplegia, exophthalmos, amblyopia, Gorner's syndrome, autumn stagnation. These symptoms last for days or weeks, after which a period of remission begins. But; some neurological symptoms were avoided. From remission that lasts for months or years, the disease can recur. MRI, carotid angiography and orbital venography reveal granulomatous inflammatory processes in the cavernous part of the cavernous sinus, fallopian tubes and internal carotid artery.

The pathogenesis of the disease is not well studied. Treatment with corticosteroids gives good results. Therefore, painful ophthalmoplegia syndrome is considered a clinical manifestation of autoimmune diseases. Similar syndrome is observed in cavernous sinus tumors, aneurysms of the cavernous part of the internal carotid artery, and basal pachymeningitis. Sphenoidal fissure syndrome (Jacob-Negri syndrome). From the cranial cavity through the upper autumn fissure (sphenoidal fissure) into the autumn bowl III, IV and VI nerves concentration g. The joint damage of ophtalmicusmmr is called sphenoidal fissure syndrome. Symptoms of the disease are observed on one side.

Jacob-Negri syndrome is a tumor or biratula sphenoidal fissure extending from the nasopharynx to the middle cranial fossa; occurs in the aneurysm of the cavernous part of the internal carotid artery. The main symptoms of this syndrome are progressive ophthalmoplegia, exophthalmos, and trigeminal pain in the forehead.

Jefferson syndrome. This syndrome develops in the aneurysm of the cavernous part of the internal carotid artery. Its main symptoms are severe pain in the fronto-orbital sac; attacks, strong noise on one side of the head, ophthalmoplegia and exophthalmos.

Garsen syndrome Unilateral damage to several cranial nerves is called Garsen syndrome. Garsen syndrome is also called hemicranial polyneuropathy because the cranial nerves are damaged on one side only.

The cranial nerves on the other side are not damaged. Garsen syndrome almost always develops in tumors that grow from the base of the skull. These are chondromas or chondrosarcomas, which grow from the pyramid of the nasopharynx, pons, or temporal bone. In Garsen syndrome, of course, the destruction of these bones is also observed.

Psychogenic pains of the face. Sharp or constant mental zurik; aches and pains that appear on the face from work are called psychogenic aches. Such pains are mainly observed in depression, neurasthenia and hysteria. Unlike true neuralgia, psychogenic pains occur on both sides of the face, do not radiate along the nerve pathways, and do not have trigger areas (whichever area of the face is pressed hurts). The patient, on the one hand, describes the pain as unbearably strong, on the other hand, says that he has not been able to find time to see a doctor since.

Melkersson-Rosenthal syndrome. The etiology is unclear. The facial nerve is damaged first on one side and then on the other. The disease is accompanied by paralysis of facial muscles, angioneurotic edema of the face, granulomatosis and cracking of the tongue surface. The disease is recurrent. The pain in the face becomes dull. Relieving facial pain Carbamazepine is used as the main drug in relieving facial pain, especially neuralgia. Even the recommendation of carbamazepine helps to compare neuralgia with painkillers. This is because carbamazepine is very effective in relieving neuralgia in Karaganda.

Carbamazepine does not stop the pain every day, but it decreases in 1-2 days. When the dose of the drug is brought to a certain amount, the pain stops or decreases sharply. Accordingly, its daily dose is determined and maintained for 4-6 weeks. Once the desired result is achieved, the dose of the drug is gradually reduced. Sometimes carbamazepine is recommended for a long time, ie 6-12 months. Carbamazepine is recommended for one of the following medications (daily dose):

- Gabapentin 300-900 mg, oxcarbamazepine 400-600 mg.

- Clonazepam 1-2 mg, phenytoin 100-300 mg.

- Voltaren 50-150 mg, ksefokam 8-16 mg, tramal 50-200 mg.

- Amitriptyline $25-75 \mathrm{mg}$ or fluoxetine $20-40 \mathrm{mg}$.

- R Nozepam 10-20 mg or sanopax 25-75 mg.

In herpetic neuralgia, immediate antiviral medications are recommended. Zovirax is administered Volume 3, Issue XI, November 2020|21 
intravenously in a dose of 250 or $500 \mathrm{mg}$ by adding $200 \mathrm{ml}$ of $0.9 \%$ sodium chloride or $5 \%$ glucose solution. Zoviraks is administered at this dose 2-3 times a day, intravenously for 5-7 days. If the pain worsens, it is necessary to add $5 \mathrm{ml}$ of $20 \%$ sodium oxybutyrate to $0.9 \%$ sodium chloride or $5 \%$ glucose solution and infuse intravenously. Antidepressants and tranquilizers are used in other types of prosopalgia, such as pain of psychogenic etiology. Antidepressants amitriptyline $25 \mathrm{mg} 2-3$ times a day or fluoxetine (portal, prozac) 20 mg 2-3 times a day, tranquilizers phenazepam $0.5 \mathrm{mg}$ or nozepam (tazepam, oxazepam) $10 \mathrm{mg}$ 1-2 times a day ordered.

Reflexology (acupuncture, hydrocortisone phonophoresis) is also widely used in the treatment of patients. In short, if these diseases are not treated in time, the outer parts of the face become crooked as a result of nerve dysfunction. If the disease is not treated in time, the disease can end in death. With this in mind, the sooner the disease is diagnosed and treated, the better the chances of recovery.

\section{REFERENCES}

1. Z. Ibodullayev "Neurology" Tashkent 2017 pp. 254-256

2. I. Muhammedov, E. Eshboyev, N.Zokirov, M.Zokirov "Microbiology Immunology Virology" Tashkent 2006 pp. 531-532

3. I. Muhammedov "Medical virology" Tashkent 2012

4. R. Haitov "Immunology" Tashkent 1996

5. https://doi.org/10.5958/2249-7137.2020.00450.4 